\title{
TRÉMATODES DE CHIROPTÈRES DE MADAGASCAR
}

\section{Identification de Plagiorcbis vespertilionis et description de trois Lecitbodendriidae nouveaux.}

\author{
par Josette RICHARD
}

Les parasites de Chiroptères qui font l'objet de cette note ont été recueillis à Madagascar par E.-R. Brygoo, A.-G. Chabaud et R. Houin qui nous ont confié le soin de les étudier. Nous les remercions très vivement.

Nous exprimons toute notre reconnaissance au Professeur G. Dubois qui a eu la très grande amabilité d'examiner ce matériel et nous a fait profiter de son expérience des Trématodes de Chiroptères.

Nous avons identifié quatre espèces, un Plagiorchiidae: Plagiorchis (Plagiorchis) vespertilionis (Müller, 1784) et trois Lecithodendriidae: Acanthatrium (Acanthatrium) houini n. sp. Papillatrium (Papillatrium) inversum n. g., n. sp., et Prosthodendrium (Prosthodendrium) chilostomum madagascariense n. subsp.

Les préparations in toto et les coupes sont déposées au Laboratoire de Zoologie (Vers) du Muséum National d'Histoire Naturelle.

\section{PLAGIORCHIS (PLAGIORCHIS) VESPERTILIONIS (Müller, 1784) Braun, 1900 (cf. fig. 1)}

Notre matériel de cette espèce provient de deux lots différents. Les hôtes et les localisations géographiques sont les suivants :

Eptesicus pusillus (Lecomte), capturé à Tananarive en septembre 1960; une dizaine de Trématodes (lot $104 \mathrm{Z}$; préparations $236 \mathrm{~TB}$ à $243 \mathrm{~TB}$ ).

Eptesicus matroka (Th. et Schw.), apporté à l'Institut Pasteur de Tananarive par le $\mathrm{D}^{\mathrm{r}}$ Radaody-Ralarosy en octobre 1957. Deux Trématodes localisés dans J'intestin (lot $180 \mathrm{Z}$; préparation $15 \mathrm{TC}$ ). 
Nous ne donnerons pas une description complète de cette espèce. Signalons seulement que nous avons constaté des variations morphologiques importantes: la limite antérieure des vitellogènes varie depuis le niveau postérieur de l'acétabulum jusqu’à
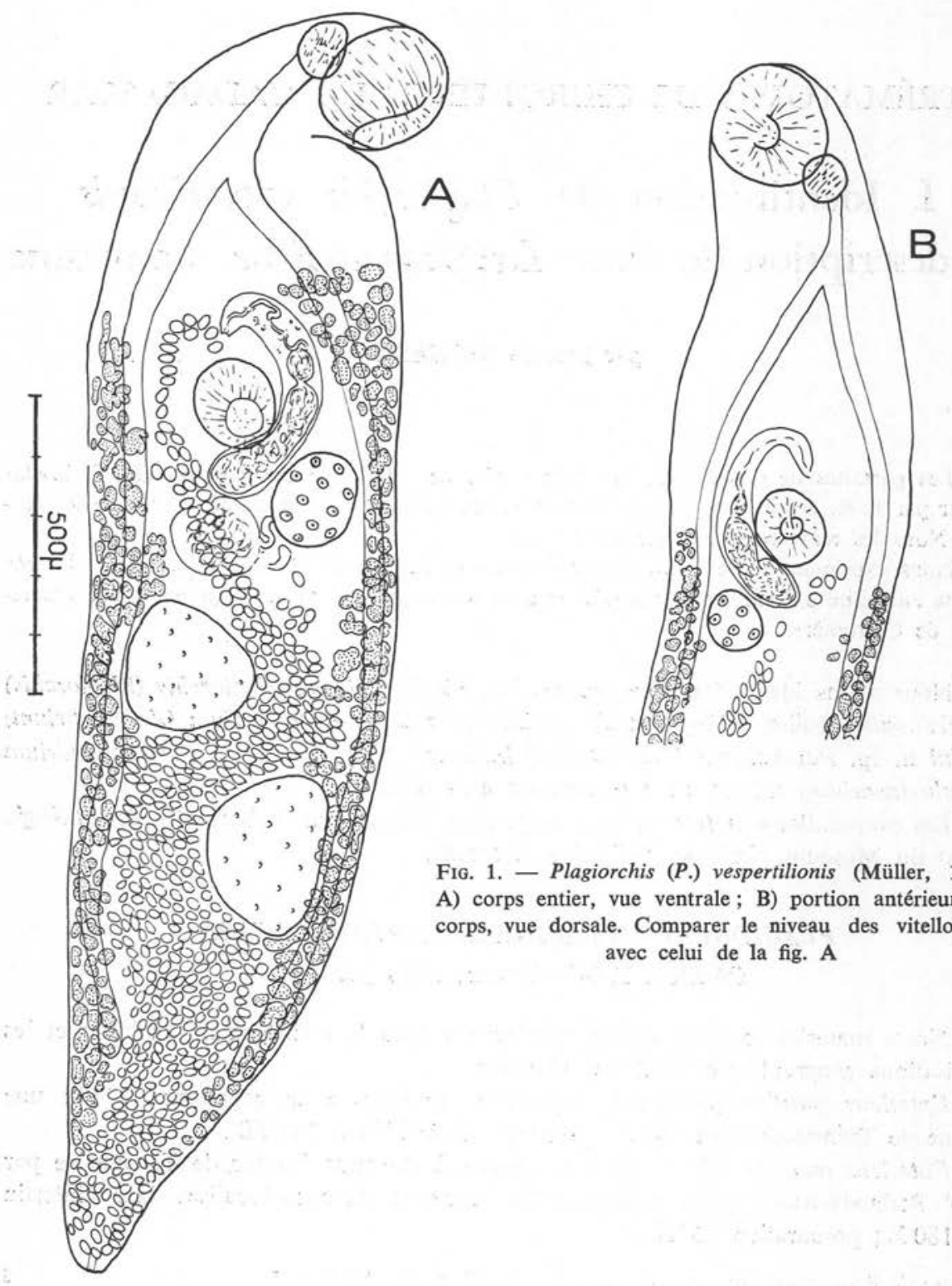

Fı́. 1. - Plagiorchis (P.) vespertilionis (Müller, 1784). A) corps entier, vue ventrale; B) portion antérieure du corps, vue dorsale. Comparer le niveau des vitellogènes avec celui de la fig. A 
un niveau très antérieur à l'orifice génital (cf. fig. 1). En outre, la poche du cirre est plus ou moins coudée en avant de l'acetabulum; ce coude est situé à des niveaux très différents.

DIMENSIONS EXTRÊMES DES SPÉCIMENS DU LOT $104 \mathrm{z}$ :

\begin{tabular}{|c|c|}
\hline ongueur $\ldots$ &, $30 \mathrm{~mm}$ \\
\hline Largeur & $400-570 \mu$ \\
\hline entouse buccale & $200-215 / 175-245$ \\
\hline harynx & $65-95 / 75-110 \mu$. \\
\hline isc & $0 \mu$. \\
\hline $\mathrm{m}$ & $70 / 140-175$ \\
\hline Ova & $165-$ \\
\hline . & $215-2$ \\
\hline sstérieur ... & $260-325 / 200-220$ \\
\hline$\ldots$ & $450-575 / 75-80$ \\
\hline D. & $32-34 / 17-20 \mu$. \\
\hline
\end{tabular}

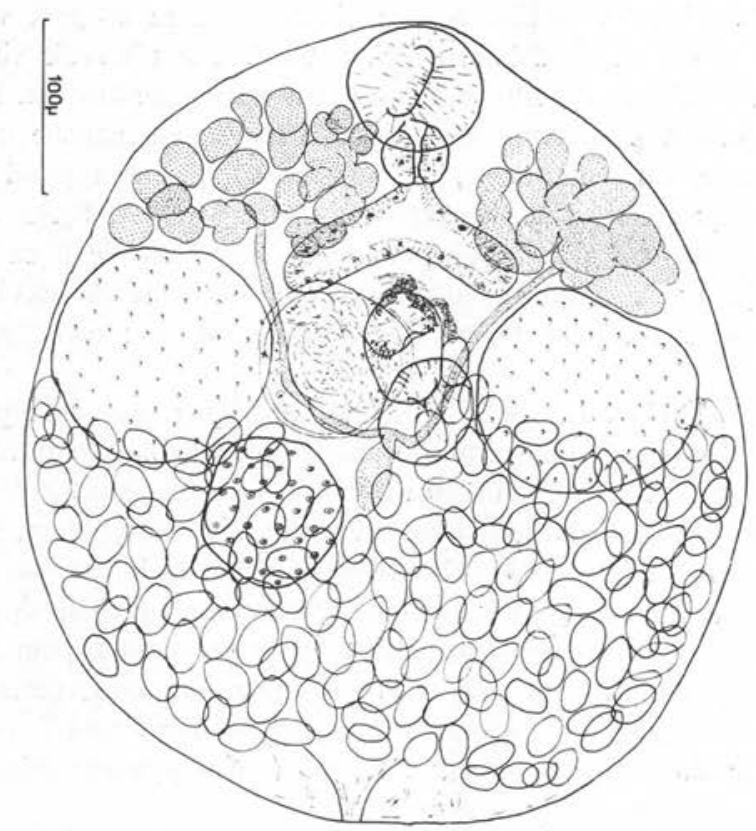

FIg. 2. - Acanthatrium (A.) houini n. sp.: corps entier, vue ventrale 


\section{ACANTHATRIUM (ACANTHATRIUM) HOUINI n. sp.}

Un seul lot $(171 \mathrm{Z})$ de ce parasite nous a été remis par R. Houin. Il comprend 70 à 80 individus recueillis dans le duodénum d'une chauve-souris indéterminée capturée à Ranomafana (novembre 1964). Les préparations sont numérotées de 1 TC à 19 TC.

Description (cf. fig. 2).

Le corps petit, ovale à circulaire, est aplati dorso-ventralement et mesure $310-490 / 320-430 \mu$. Nous n'avons pas observé d'épine sur la cuticule. La ventouse buccale, subterminale, mesure 45-67/65-81 $\mu$; l'acétabulum, équatorial, est plus petit et mesure 45-50/48-58 $\mu$; il est distant de l'extrémité antérieure du corps de 150-180 $\mu$. Le pharynx mesure 24-32/27-40 $\mu$; l'œsophage a environ 20-30 $\mu$ de longueur; les caecums, très courts, 50-60 $\mu$, se terminent tangentiellement aux testicules. La vessie excrétrice est en forme de V.

Appareil génital femelle: L'ovaire, dextre, postacétabulaire, est grossièrement sphérique ; non lobé, il mesure 70-115/65-90 $\mu$. La glande de Mehlis et le réceptacle séminal situés à gauche et en arrière de l'ovaire sont difficilement visibles dans les préparations in toto. Le canal de Laurer débouche dorsalement; il est bien visible dans les coupes transversales correspondant au niveau de l'orifice génital. Les vitellogènes précaecaux sont composés de follicules (15 à 20) plus ou moins discernables les uns des autres selon les spécimens; les vitelloductes se dirigent vers l'arrière en passant tangentiellement au bord interne des testicules; le réservoir vitellin, là où les deux vitelloductes confluent, est situé en avant et à gauche de l'ovaire. L'utérus occupe presque toute la moitié postérieure du corps; il remonte à gauche de l'acetabulum. Sa portion terminale, ou métraterme, a un tracé sinueux; sa paroi d'environ $3 \mu$ d'épaisseur a un aspect strié dans les préparations in toto (cf. fig. 3 A); il s'oriente vers la droite en contournant la masse prostatique dans laquelle il ne pénètre pas, et s'unit au canal éjaculateur immédiatement après la sortie de ce dernier de la masse prostatique (cf. fig. $3 \mathrm{~B}$ ), pour former le sinus génital. Les œufs mesurent 28-32/ 16-19 $\mu$.

Appareil génital mâle: Les deux testicules sphériques, para- ou préacétabulaires, sont volumineux, plus grands que la masse prostatique ; ils mesurent $72-130 / 70-115 \mu$ pour le testicule droit, et $65-140 / 90-130 \mu$ pour le testicule gauche. La masse prostatique est située entre les deux testicules, en avant de l'acétabulum. Plus ou moins circulaire, elle mesure 60-90;65-95 $\mu$. La vésicule séminale interne est pelotonnée sur elle-même ; les cellules prostatiques volumineuses entourent le canal éjaculateur. Canal éjaculateur et utérus s'unissent en dehors de la masse prostatique pour former le sinus génital (1). Celui-ci aboutit dans une cavité ventrale du corps, l'atrium génital (cf. fig. $3 \mathrm{~A}$ ), qui abrite également l'acétabulum et s'ouvre largement à l'extérieur. Latéralement à l'orifice sinusal, l'atrium présente deux lobes spinulés étirés dorso-ventra-

(1) Selon les indications de G. Dubois (in litt., 22 mars 1966), nous n'utiliserons pas le terme « atrium bipartite » cf. Dubois, 1960, p. 52 et 1961, p. 276); avec cet auteur, nous désignerons, par "sinus génital », la petite cavité au fond de laquelle «on reconnaît les orifices séparés des conduits vecteurs mâle et femelle» (cf. Looss, 1896, p. 66), et par \& atrium», la cavité ventrale largement ouverte à l'extérieur dans laquelle aboutit le sinus. 


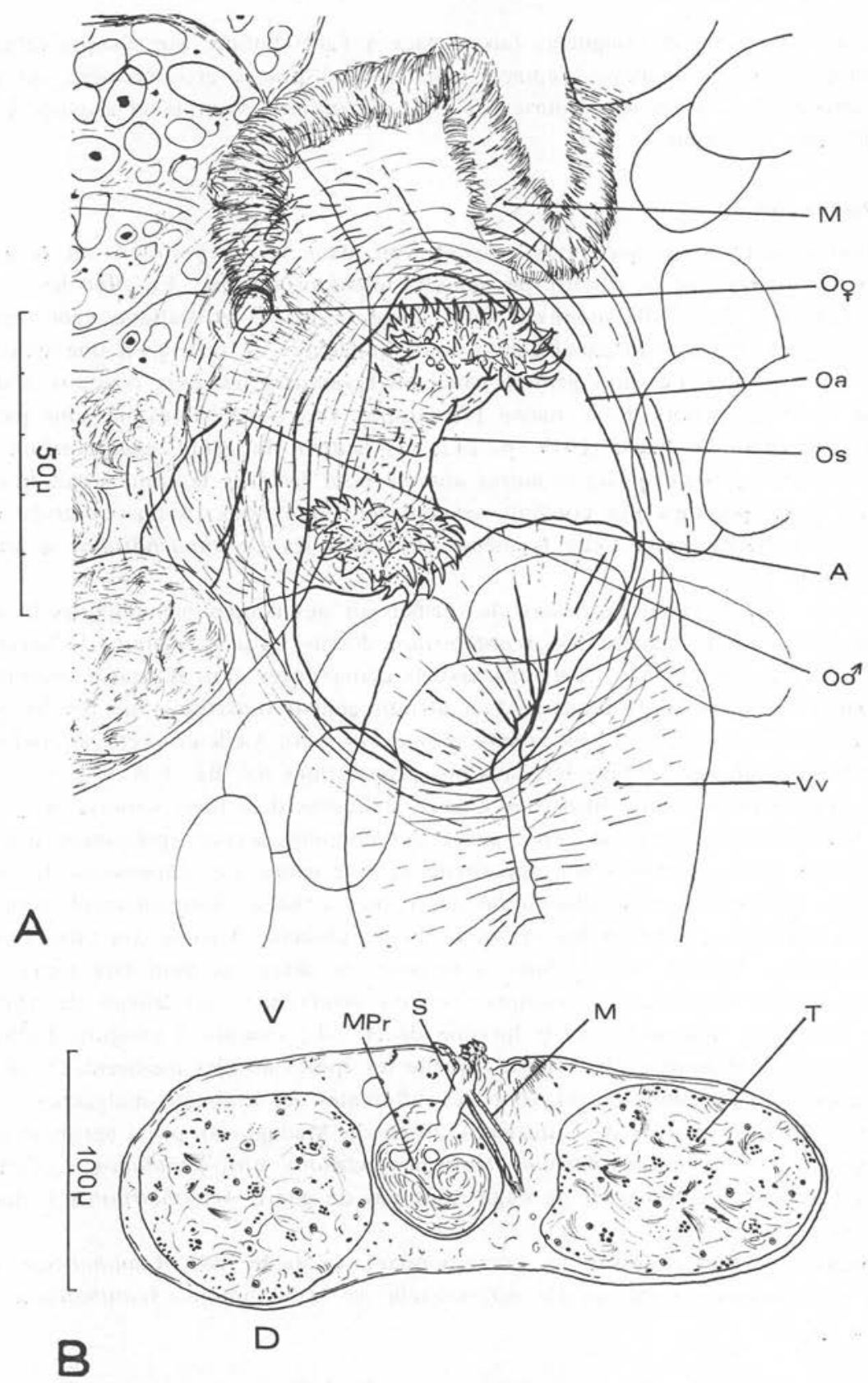

Fig. 3. - Acanthatrium (A.) houini n.sp. A) détail des parties terminales de l'appareil génital, vue ventrale; B) coupe transversale (7,5 $\mu$ d'épaisseur), au niveau du sinus génital 
lement, de 30 à $40 \mu$ de longueur, faisant face à l'acétabulum. Sur chaque lobe, on peut compter une soixantaine d'épines; elles sont disposées grossièrement sur cinq arcs ventro-dorsaux. Elles sont courtes ( $5 \mu$ au maximum), pointues et arquées à leur extrémité vers l'extérieur.

\section{Discussion.}

Contrairement à ce que nous croyons voir dans les préparations in toto (cf. fig. $3 \mathrm{~A}$ ), le métraterme ne pénètre pas dans la masse prostatique. En effet, les coupes histologiques (cf. fig. 3 B) montrent qu'il longe la masse prostatique ventralement sans y pénétrer et s'unit au canal génital mâle en dehors de celle-ci, à une vingtaine de $\mu$ du pore génital. Le sinus génital, canal où fusionnent les deux conduits sexuels, est donc situé en dehors de la masse prostatique. Ces observations ne sont pas en accord avec celles de Faust (1919, p. 212) (1), auteur du genre Acanthatrium, sur A. (A.) nycteridis, et de quelques autres auteurs pour lesquels le sinus serait interne. Peut-être la morphologie des conduits sexuels, telle que nous l'avons observée chez les spécimens malgaches, est-elle la même chez tous les Lecithodendriidae à atrium génital spinulé...

A notre avis, il serait nécessaire de vérifier sur des coupes histologiques la morphologie des conduits sexuels des Acanthatrium décrits jusqu'à ce jour: le caractère (cf. G. Dubois, 1960, p. 52) « conduits sexuels convergeant dans la masse prostatique pour déboucher dans la profondeur d'un atrium génital bipartite \$, ne résulte peutêtre que de l'observation de spécimens montés in toto. Celle-ci prête à confusion comme nous avons pu le constater dans nos préparations (cf. fig. $3 \mathrm{~A}$ ).

En conséquence, nous admettrons que ces parasites de Chau res-souris malgaches qui, à l'exception du point de convergence des conduits sexuels, présentent tous les caractères du genre Acanthatrium, appartiennent à ce genre. Les dimensions du corps, le nombre, la longueur et la disposition des épines atriales, essentiellement, sont des caractères constants chez tous les individus du lot observé. Aucune des onze espèces retenues par G. Dubois (1961), dans sa révision du genre, ne peut être rapprochée de ce parasite; les espèces contemporaines ou postérieures au travail de Dubois, A. (A.) rotundum Watanabe, 1959 [proche de $A$. (A.) ovatum Yamaguti, 1939], et A. (A.) lunatum Williams, 1960 (chez laquelle les épines atriales mesurent 15-28 $\mu$ et sont groupées en une seule plage), sont très différentes des individus malgaches.

Enfin, chez le parasite de Caméléons décrit de Madagascar par Capron et coll., 1961, sous le nom «Acanthatrium (Prosthodendrium) atrio-papillatum », l'atrium génital est dépourvu d'épines. Il ne s'agit donc pas du genre Acanthatrium (cf. discussion p. 8).

L'espèce décrite ci-dessus ne correspondant à aucun des Acanthatrium déjà décrits, nous pensons donc qu'elle est nouvelle et la nommons Acanthatrium $(A$.) houini n. sp.

(1) «The uterus enters the genital atrium to the left of the ejaculatory duct... The prostate glands surrounding the metraterm and opening into the ejaculatory duct consists of a large spherical mass of unicellular glands. » 


\section{PAPILLATRIUM (PAPILLATRIUM) INVERSUM n. g., n. sp.}

Les deux lots de ce parasite proviennent des chauves-souris suivantes: Eptesicus pusillus (Lecomte), Tananarive (août 1960), dont l'intestin contenait un spécimen
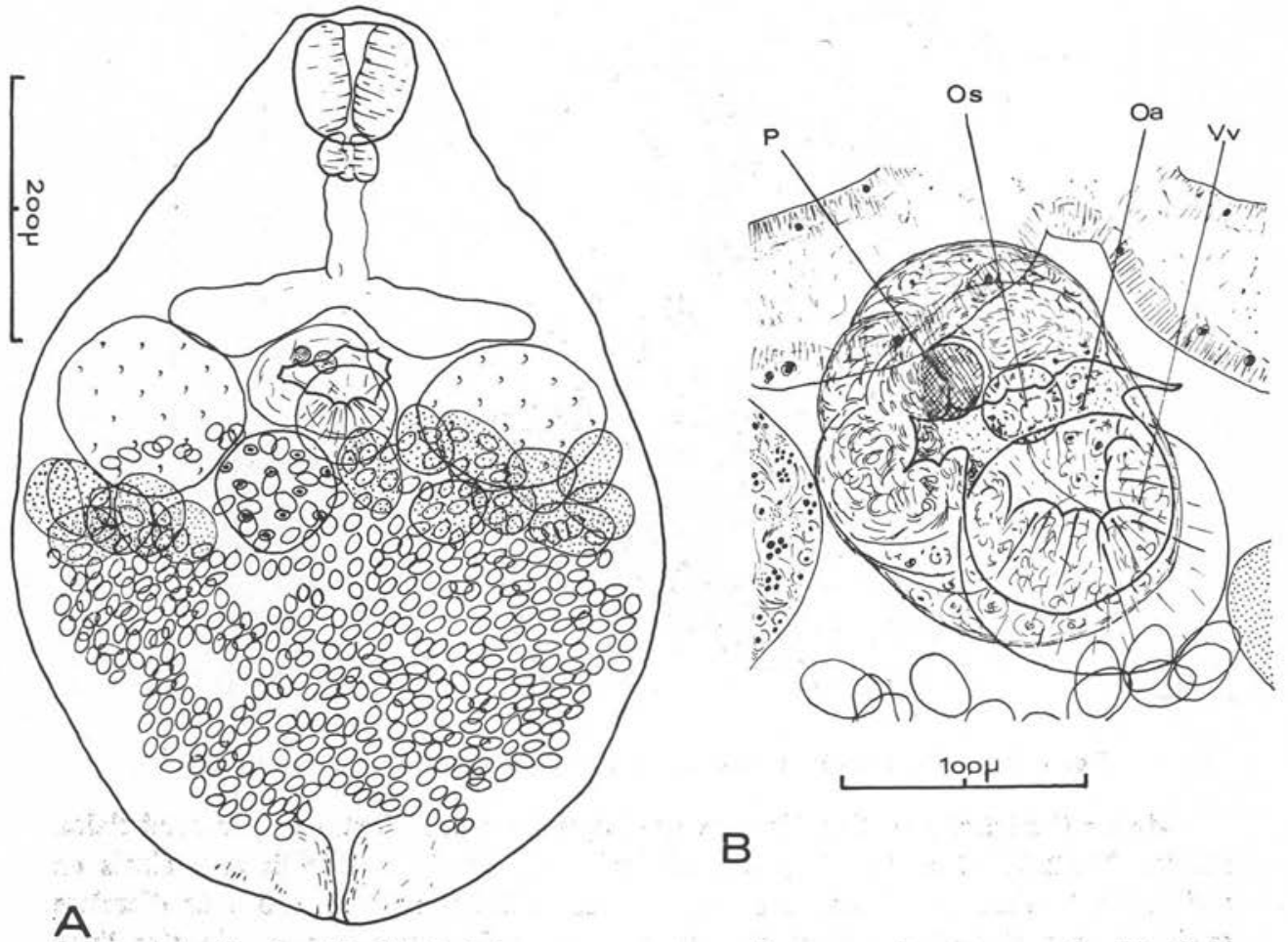

Fig. 4. - Papillatrium (Papillatrium) inversum n. g., n. sp. A) corps entier, vue ventrale ; B) détail des parties terminales de l'appareil génital, vue ventrale

(lot $104 \mathrm{Z}$, préparation $\mathrm{n}^{\circ} 30 \mathrm{TC}$ ), et Chaerephon limbatus (Peters), capturé dans le grenier de la maison forestière à Périnet (mars 1961) dont l'intestin était parasité par une quarantaine de Trématodes (lot $179 \mathrm{Z}$, préparation $20 \mathrm{TC}$ à $28 \mathrm{TC}$ ).

Description (cf. fig. 4. Lot 179z).

Le corps piriforme, aplati dorso-ventralement, mesure 620-810 $\mu$ de longueur et $400-490 \mu$ de largeur. Nous n'avons pas observé d'épines cuticulaires. La ventouse buccale subterminale mesure 64-86/72-98 $\mu$. L'acétabulum, équatorial, est subégal à la ventouse buccale et mesure 60-85/60-93 $\mu$; son bord antérieur est situé à 220-300 $\mu$ de l'extrémité antérieure. Le pharynx mesure 26-40/37-48 $\mu$. L'œsophage est court (environ $50 \mu$ ), les caecums également courts mesurent $150 \mu$. La vessie excrétrice est en forme de $\mathrm{V}$. 


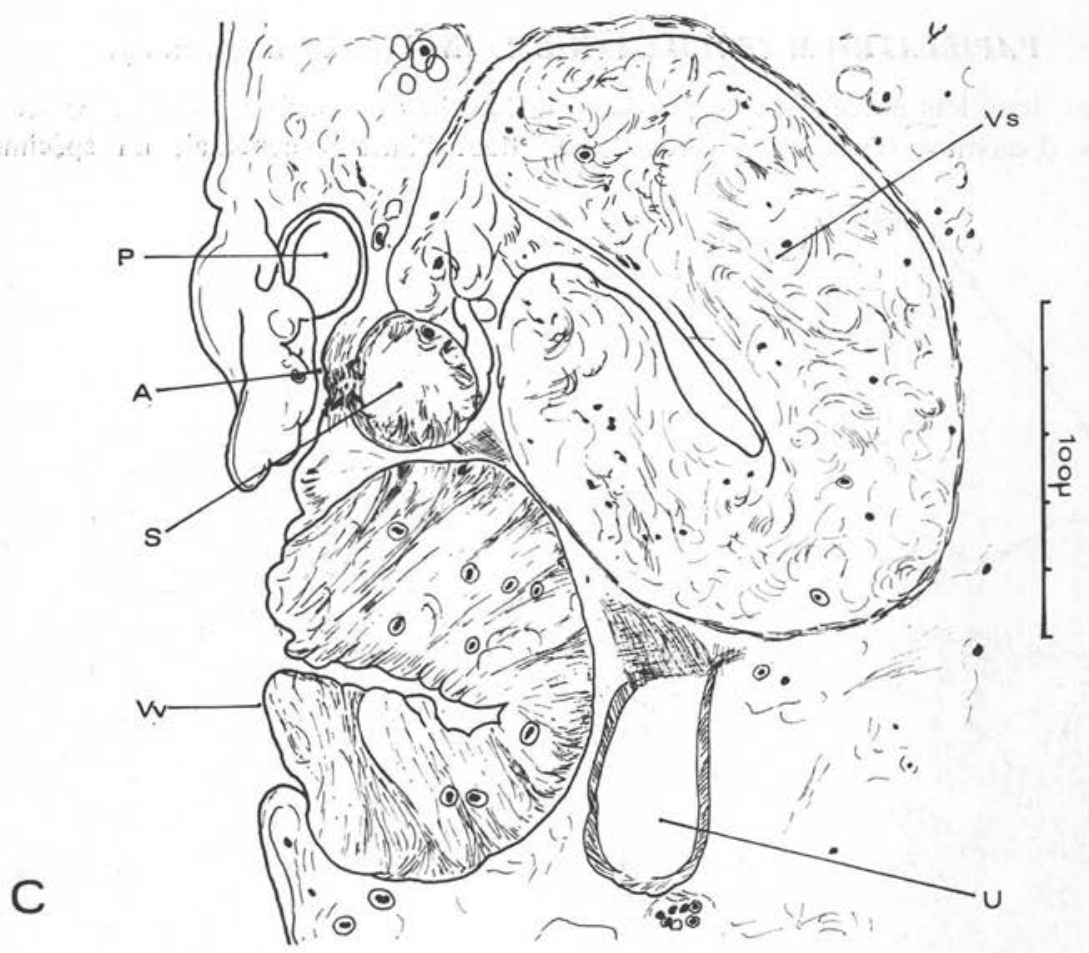

FIg. 4 bis. - C) coupe longitudinale $(7,5 \mu$ d'épaisseur) au niveau de la papille

Appareil génital femelle: L'ovaire sphérique ou ovale, dextre et paracétabulaire, mesure 75-92/75-97 $\mu$. Le réceptacle séminal et la glande de Mehlis sont situés en arrière de l'ovaire. Les vitellogènes composés de follicules arrondis $(50 \mu$ de diamètre environ), sont disposés en deux grappes plus ou moins transversales, séparées l'une de l'autre par l'ovaire, et situées juste en arrière des testicules. On compte 9 follicules à gauche, et 7 à droite (1). Chez un spécimen anormal, nous en avons compté 10 à gauche et 5 à droite. L'utérus occupe toute la partie postérieure du corps en arrière de l'acétabulum; il est rempli d'œufs qui mesurent 22-25/13-15 $\mu$.

Le métraterme remonte soit à gauche de l'acétabulum, soit dorsalement par rapport à celui-ci dans l'axe sagittal, longe la masse prostatique et s'ouvre dans le sinus génital qui est préacétabulaire et médian. Dans l'atrium génital (v. ci-dessus, note p. 4) (cf. fig. $4 \mathrm{~B}$ et $\mathrm{C}$ ), et légèrement à droite, se trouve une papille ovoïde de 22-26/ 13-17 $\mu$, dont le grand axe est antéro-postérieur; elle est rattachée à la paroi de l'atrium par son bord latéral droit. L'ouverture de l'atrium est large, irrégulièrement Tobée ou découpée (cf. fig. 4 B).

(1) Chez Lecithodendrium (L.) linstowi Dollfus, 1931, ces chiffres sont inversés : on compte, en effet, neuf follicules à droite et sept à gauche. C'est pour cette raison que nous nommons notre espèce inversum. 
DIMENSIONS DU SPÉCIMEN $104 \mathrm{z}$ :

\begin{tabular}{|c|c|}
\hline Longueur & $1,42 \mathrm{~mm}$ \\
\hline Largeur & $685 \mu$ \\
\hline Ventouse buccale $\ldots \ldots \ldots \ldots \ldots$ & $98 / 132 \mu$ \\
\hline Pharynx $\ldots \ldots \ldots \ldots \ldots \ldots \ldots \ldots$ & $37 / 52$ \\
\hline Esophage $\ldots \ldots \ldots$ & $125 \mu$ \\
\hline 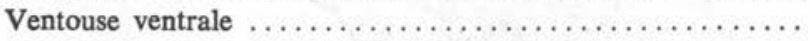 & $117 / 102$ \\
\hline 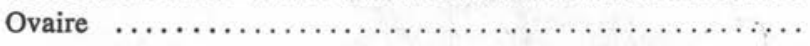 & $175 / 140$ \\
\hline 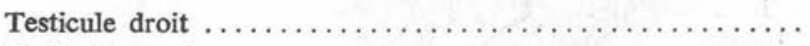 & $225 / 100$ \\
\hline Testicule gauche $\ldots \ldots \ldots \ldots \ldots \ldots$ & $200 / 155$ \\
\hline CEufs & $25 / 13$ \\
\hline
\end{tabular}

Appareil génital mâle: Les deux testicules volumineux, sphériques, para- ou préacétabulaires, sont tangents à l'extrémité des caecums ; ils mesurent 90-125/100-130 $\mu$ pour le testicule droit, et 100-125/125-130 $\mu$ pour le testicule gauche. La masse prostatique, plus petite que les testicules, est située en avant de l'acétabulum ; elle mesure $75 / 100 \mu$ et contient une vésicule séminale pelotonnée sur elle-même et des cellules prostatiques. Il n'y a pas de cirre.

\section{Discussion.}

Quand on se rapporte aux descriptions des espèces des genres Lecithodendrium Looss, 1896, et Prosthodendrium Dollfus, 1931, on remarque qu'aucune d'entre elles ne mentionne de papille atriale du type observé chez les parasites ci-dessus décrits. Parallèlement aux groupes d'espèces (cf. G. Dubois, 1960, tableau p. 52) à atrium génital spinulé, d'une part, et à atrium génital inerme, d'autre part (1), nous avons donc un troisième groupe de parasites dont l'atrium génital est muni d'une papille. Nous serions tentée d'accorder une bonne valeur à ce caractère, d'autant plus qu'il paraît particulier aux espèces malgaches, mais la découverte d'autres espèces permettra seule d'affirmer ou d'infirmer cette considération accessoire (cf. fig. 6).

Nous créons donc un nouveau genre Papillatrium dont le type est Papillatrium inversum; la diagnose est la suivante :

Lecithodendriinae : corps petit, ovale, circulaire ou piriforme plus long que large, à cuticule non spinulée, à ventouse buccale plus grande que l'acétabulum ou subégale, à ovaire submédian, dextre, postacétabulaire et non lobé, à vitellogènes postesticulaires, à testicules para- ou préacétabulaires, à conduits sexuels débouchant dans un sinus s'ouvrant lui-même dans un atrium muni d'une papille.

Nous ne connaissons actuellement dans ce genre sensu stricto que notre espèce inversum parasite de l'intestin de Chiroptères. Nous devons cependant signaler que l'espèce nommée par Capron, Deblock et Brygoo (1961), Acanthatrium (Prosthodendrium atrio-papillatum et décrite de Caméléons malgaches, n'appartient pas au genre Acanthatrium; sa papille charnue rappelle l'espèce inversum (cf. fig. 5). Cependant, les vitellogènes sont prétesticulaires (2) et les hôtes sont des Caméléons. Pour cette raison,

(1) Nous ne retenons que ce caractère différentiel entre les deux groupes d'espèces, car nous avons vu plus haut (cf. p. 418) que le caractère «conduits sexuels convergeant dans la masse prostatique » serait à vérifier par études histologiques.

(2) Les follicules vitellogènes droits et gauches fusionnent dorsalement sur le plan sagittal. 


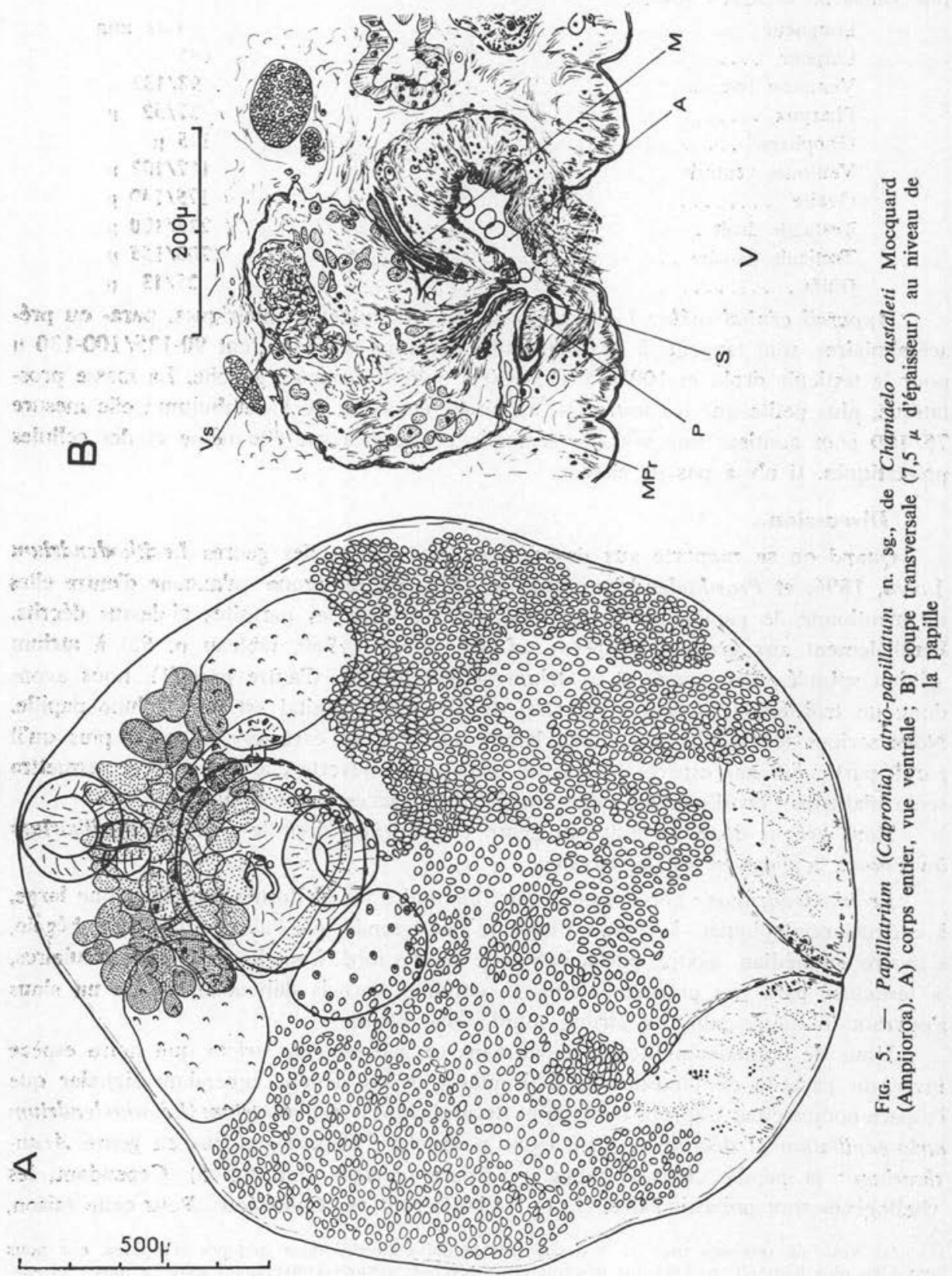


la position de ce parasite demeure quelque peu énigmatique. Nous proposons pour elle le sous-genre Capronia que nous considérons, au moins présentement, comme un sous-genre de-Papillatrium.

\section{PROSTHODENDRIUM (PROSTHODENDRIUM) CHILOSTOMUM MADAGASCARIENSE n. subsp.}

Nous avons deux lots de ce parasite $(178 \mathrm{Z}$ et $179 \mathrm{Z})$. Ils ont été récoltés chez deux chauves souris de l'espèce Chaerephon limbatus (Peters) capturées à Périnet (mars 1961) (préparations 43-47 Tc).

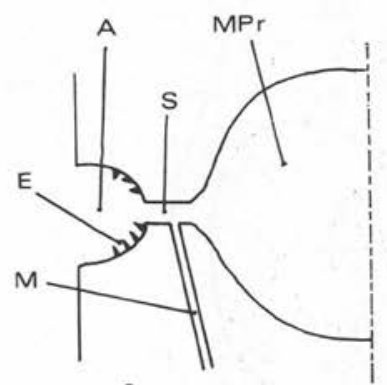

A

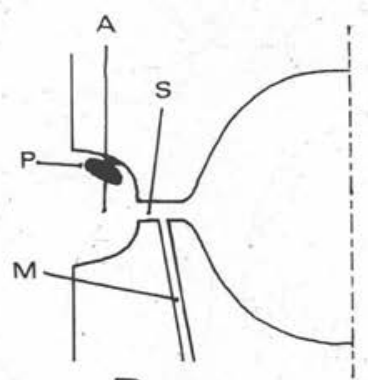

B

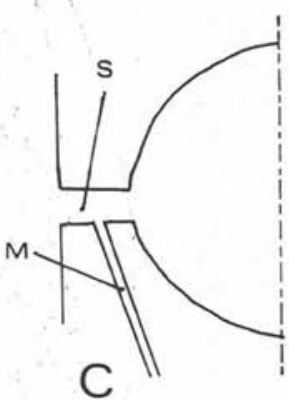

Fig. 6. - Schéma de la morphologie des conduits sexuels. A) Acanthatrium et Mesothatrium; B) Papillatrium ; C) Prosthodendrium et Lecithodendrium

\section{Description (cf. fig. 7).}

Le corps ovale à circulaire est couvert de très petites épines de 6 à $8 \mu$ de longueur. Il mesure 620-900/470-690 $\mu$. La ventouse buccale subterminale est très volumineuse; elle mesure 145-230/195-220 $\mu$. L'acétabulum situé à environ $60 \mu$ du bord postérieur de la ventouse buccale est également volumineux. Il mesure 130-170/155-197 $\mu$. Le rapport des dimensions ventouse buccale/acétabulum varie de 1,15 à 1,40 . Le pharynx est petit, 65-85/65-77 $\mu$. L'œsophage est court, plus ou moins récurrent. Les caecums très courts, 150-200 $\mu$, n'atteignent pas les testicules. La vessie excrétrice est en forme de V.

Appareil génital femelle: L'ovaire volumineux, ovale à circulaire, est dextre. Situé dorsalement par rapport à la ventouse buccale et légèrement à droite du plan sagittal, il mesure 120-187/82-150 $\mu$. Les vitellogènes dorsaux, précaecaux, sont formés de deux grappes de follicules arrondis situés de part et d'autre de la ventouse buccale. On compte 9 follicules à droite et 7 à gauche. Les vitelloductes passent juste en arrière du pharynx. L'utérus sinueux est très développé ; il recouvre l'hémisphère postérieur des testicules et de l'acétabulum. Les œufs mesurent 24-26/14-15 $\mu$. 
Appareil génital mâle: Les deux testicules sont en position para- ou préacétabulaire et mesurent 200/200-212 $\mu$ pour le testicule droit, et 187-210/165-175 $\mu$ pour le testicule gauche. La masse prostatique, para- ou préacétabulaire, sphérique, est plus

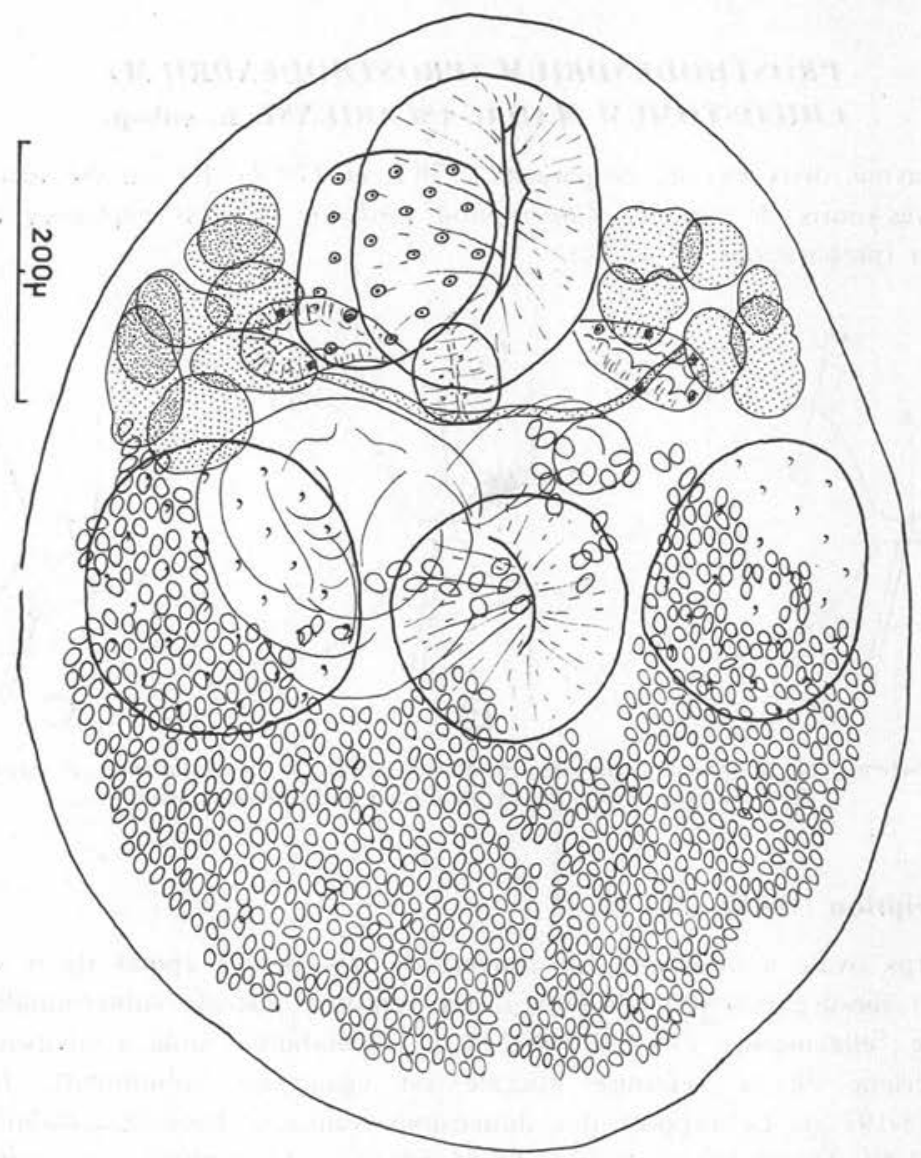

FIG. 7. - Prosthodendrium (P.) chilostomum madagascariense n. subsp.: corps entier, vue ventrale

grande que les testicules. Les deux conduits sexuels mâle et femelle débouchent dans un simple sinus qui s'ouvre à l'extérieur au niveau d'un pore situé en avant de l'acétabulum et à gauche du plan sagittal.

\section{Discussion.}

Ce Trématode appartient au genre Prosthodendrium (sous-genre Prosthodendrium) Dollfus, 1931. Ses caractères morphologiques concordent avec ceux de $P$. $(P$.) chilostomum (Mehlis, 1831). Comme chez cette espèce, l'ovaire est situé « in den 
Seiten des Halses » (Mehlis, 1831, p. 187), la ventouse buccale volumineuse représente le quart de la longueur du corps, les testicules sont latéraux par rapport à la ventouse ventrale; les œufs ont les dimensions indiquées par les auteurs. Elle possède 9 follicules vitellogènes à droite, et 7 à gauche. Cependant l'acétabulum est relativement beaucoup plus grand que chez $P$. $(P$.) chilostomum tel qu'il est redécrit par Dubois, 1955 et 1960 , et K. Odening, 1964 (cf. tableau I).

Comparaison des données relatives aux ventouses chez les auteurs

\begin{tabular}{|c|c|c|c|c|}
\hline & Dubois, 1955 & Dubois, 1960 & Odening, 1964 & Richard \\
\hline Localisation & Intestin & Intestin & $\begin{array}{l}\text { a) Vésicule } \\
\text { biliaire } \\
\text { b) Intestin }\end{array}$ & Intestin \\
\hline Hôtes & $\begin{array}{l}\text { Rhinolophus } \\
\text { hipposideros }\end{array}$ & $\begin{array}{l}\text { Miniopterus } \\
\text { schreibersi } \\
\text { schreibersi } \\
\text { et } \\
\text { Nyctalus noctula } \\
\text { noctula }\end{array}$ & Myotis myotis & $\begin{array}{l}\text { Chaerephon } \\
\text { limbatus }\end{array}$ \\
\hline $\begin{array}{l}\text { Ventouse } \\
\text { buccale }\end{array}$ & $160-183 / 110-146$ & $150-235 / 110-160$ & $\begin{array}{l}\text { a) } 138-166 / 152- \\
143 \\
\text { b) } 145-183 / 141- \\
176\end{array}$ & $145-230 / 195-220$ \\
\hline $\begin{array}{l}\text { Ventouse } \\
\text { ventrale }\end{array}$ & $85-96 / 94-105$ & $81-118 / 96-125$ & $\begin{array}{l}\text { a) } 90-100 / 83-104 \\
\text { b) } 86-104 / 86-100\end{array}$ & $130-170 / 155-195$ \\
\hline
\end{tabular}

a) Vésicule biliaire. b) Intestin.

Chez les spécimens décrits par $\mathrm{M}^{\mathrm{me}}$ J. Hurkova (1963, p. 265, fig. 3 A), le diamètre longitudinal de la ventouse buccale est 1,7 fois supérieur à celui de la ventouse ventrale, pour le même diamètre transversal (environ $140 \mu$ ), alors que ces diamètres diffèrent très peu chez nos spécimens. Nous remarquons également une forte différence entre les diamètres des deux ventouses chez le spécimen représenté par Rysavy (1956, fig. 4) ; d'après l'échelle, leurs dimensions sont approximativement les suivantes: $191 / 138 \mu$ pour la ventouse buccale, et $99 / 99 \mu$ pour l'acétabulum (1).

Chez $P$. (P.) erhardovae décrit par Rysavy (1954) l'acétabulum est également grand par rapport à la ventouse buccale mais l'ovaire est postacétabulaire et les œufs sont beaucoup plus grands (30-36/15-19 $\mu$ ).

En raison des dimensions des ventouses, nous considérons le parasite décrit ci-dessus comme une sous-espèce de $P$. (P.) chilostomum (Mehlis, 1831) et le nommons $P$. (P.) chilostomum madagascariense nov. subsp.

(1) Ces dimensions ne correspondent pas à celles indiquées dans le texte. 


\section{Résumé}

1) Plagiorchis (P.) vespertilionis (Müller, 1784) chez Eptesicus pusillus et Eptesicus matroka.

2) Prosthodendrium (P.) chilostomum madagascariense n. subsp. de Chaerephon limbatus, dont la ventouse ventrale est relativement beaucoup plus grande que celle de l'espèce type. Cette nouvelle sous-espèce ne peut être confondue avec $P$. (P.) erhardovae Rysavy, 1954, qui possède également une grande ventouse ventrale, mais dont l'ovaire est postacétabulaire.

3) Acanthatrium (A.) houini n. sp., caractérisé par ses petites épines atriales groupées sur deux éminences. Des coupes transversales montrent que les conduits génitaux mâle et femelle convergent dans un sinus situé en dehors de la masse prostatique. Il est possible que ce caractère soit celui de tous les Acanthatrium et nous pensons qu'il serait utile de le vérifier sur coupes histologiques.

4) Papillatrium (P.) inversum n.g., n. sp., d'Eptesicus pusillus et Chaerephon limbatus. Parallèlement au genre Acanthatrium caractérisé par ses épines atriales, le nouveau genre Papillatrium est caractérisé par la présence d'une papille atriale. Le type est Papillatrium (P.) inversum.

Un nouveau sous-genre Capronia est créé pour l'espèce décrite sous le nom «d'Acanthatrium (P.) atrio-papillatum» par Capron, Deblock et Brygoo (1961), parasite de Caméléons, et chez lequel les vitellogènes sont prétesticulaires.

SIGNIFICATION DES LETTRES

$\begin{array}{llll}\text { A } & \text { atrium } & \text { Oơ }^{*} & \text { orifice mâle } \\ \text { D } & \text { dorsal } & \text { P } & \text { papille } \\ \text { E } & \text { épines } & \text { S } & \text { sinus } \\ \text { M } & \text { métraterme } & \text { T } & \text { testicule } \\ \text { MPr } & \text { masse prostatique } & \text { U } & \text { utérus } \\ \mathrm{Oa} & \text { orifice de l'atrium } & \text { V } & \text { ventral } \\ \text { Os } & \text { orifice du sinus } & \text { Vv } & \text { ventouse ventrale } \\ \text { O } & \text { orifice femelle } & \text { Vs } & \text { vésicule séminale }\end{array}$




\section{Bibliographie}

CAPron (A.), Deblock (S.), Brygoo (E. R.), 1961. - Miscellanea helminthologica madagas. cariensia. Trématodes de Caméléons de Madagascar. Arch. Inst. Pasteur de Madagascar, 29, (1), pp. 1-71.

Dubors (G.), 1955. - Les Trématodes de Chiroptères de la collection Villy Aellen. Rev. suisse Zool., 62 (3), pp. 469-506.

DuboIs (G.), 1960. - Contribution à l'étude des Trématodes de Chiroptères. Révision du sous-genre Prosthodendrium Dollfus, 1931 et des genres Lecithodendrium Looss, 1896 et Pycnoporus Looss, 1899. Rev. suisse Zool., 67 (1), pp. 1-80.

FAUST (E. C.), 1919. - A new trematode, Acanthatrium nycteridis, nov. gen., nov. spec., from the little brown bat. Trans. Amer. micr. Soc. Menasha, 38 (3), pp. 209-215.

Hurkova (J.), 1963. - Bat trematodes in Czechoslovakia. I. A systematical review of occurring species. Vestn. Ceskos. Spolec. Zool., 27 (4), pp. 250-276.

Looss (A.), 1896. - Recherches sur la faune parasitaire de l'Egypte. Première partie. Mém. Inst. egypt., 3, pp. 1-252, pls 1-16, fig. 1-193.

MACY (R. W.), 1936. - Three new Trematodes of Minnesota bats with a key to the genus Prosthodendrium. Trans. Amer. micr. Soc. Menasha, 55 (3), pp. 352-359.

Mehlis (E.), 1831. - Novae observationes de entozois. Isis (Oken), (2), pp. 166-199.

Odening (K.), 1964. - Exkretionssystem und systematische Stellung einiger Fledermaustrematoden aus Berlin und Umgebund nebst Bemerkungen zum lecithodendrioiden Komplex. Z. f. Parasitenkunde, 24, pp. 453-483.

RYSAVY (B.), 1954. - (Contribution to the study of the trematodes of Rhinolophidae in our country). Vestnik Ceskoslov. Zool. Spolec. Praze, 17, pp. 298-300.

RYSAVY (B.), 1956. - (Parasitische Würmer des Fledermäuse (Chiroptera) in der Tschecho slowakei). Cesk. Parasitol., 3, pp. 161-179.

Watanabe (A.), 1959. - Studies on trematode parasites of bats in Hiroshima Prefecture, with some reference to the function of Laurer's canal. I. Japan. J. of Parasitol., 8 (6), pp. 849-957.

Williams (R. R.), 1960. - Acanthatrium lunatum n. sp. a parasite of the big brown bat and a key to the described species of Acanthatrium (Trematoda: Lecithodendriidae). Ohio J. of Sci., 60 (6), pp. 323-326.

Laboratoire de Zoologie (Vers). Muséum National d'Histoire Naturelle, 57, rue Cuvier, F-75 - Paris, 5 e. 